PHYSICAL REVIEW D 92, 125034 (2015)

\title{
Exact effective couplings of four dimensional gauge theories with $\mathcal{N}=2$ supersymmetry
}

\author{
Vladimir Mitev ${ }^{1, *}$ and Elli Pomoni ${ }^{2,3, \dagger}$ \\ ${ }^{1}$ Institut für Mathematik und Institut für Physik, Humboldt-Universität zu Berlin, \\ IRIS Haus, Zum Großen Windkanal 6, 12489 Berlin, Germany \\ ${ }^{2}$ DESY Theory Group, Notkestraße 85, 22607 Hamburg, Germany \\ ${ }^{3}$ Physics Division, National Technical University of Athens, \\ 15780 Zografou Campus, Athens, Greece \\ (Received 2 July 2014; published 30 December 2015)
}

\begin{abstract}
The anomalous dimensions of operators in the purely gluonic $\mathrm{SU}(2,1 \mid 2)$ sector of any planar conformal $\mathcal{N}=2$ theory can be read off from the $\mathcal{N}=4$ SYM results by replacing the $\mathcal{N}=4$ coupling constant with an interpolating function of the $\mathcal{N}=2$ coupling constants [1], which we refer to as the effective coupling. For a large class of $\mathcal{N}=2$ theories, we compute the weak-coupling expansion of these functions as well as the leading strong-coupling term by employing supersymmetric localization. Via Feynman diagrams, we interpret our results as the relative (between $\mathcal{N}=2$ and $\mathcal{N}=4$ ) finite renormalization of the coupling constant. Using the AdS/CFT dictionary, we identify the effective couplings with the effective string tensions of the corresponding gravity dual theories. Thus, any observable in the $\mathrm{SU}(2,1 \mid 2)$ sector can be obtained from its $\mathcal{N}=4$ counterpart by replacing the $\mathcal{N}=4$ coupling constant with the universal, for a given theory, effective coupling.
\end{abstract}

DOI: 10.1103/PhysRevD.92.125034

PACS numbers: 11.15.-q, 11.15.Tk, 11.25.Tq, 11.30.Pb

\section{INTRODUCTION AND SUMMARY}

The recent studies of $\mathcal{N}=4$ super Yang-Mills (SYM) have lead to impressive exact results and novel insights for four-dimensional gauge theories. In this paper we consider the simplest next step in four dimensions: $\mathcal{N}=2$ gauge theories. So far, exact results in gauge theories have come from using either integrability (see [2] for a review), localization [3], or a dual string theory description (AdS/CFT [4]).

The general problem of obtaining the gravity dual of $\mathcal{N}=2$ superconformal gauge theories has been studied in [5-12] with partial success. However, theories that are obtained as orbifolds of $\mathcal{N}=4 \mathrm{SYM}$ have well-known gravity duals $[13,14]$ and, in particular, the $\hat{A}_{r-1}$ quivers are dual to $\mathrm{AdS}_{5} \times S^{5} / \mathbb{Z}_{r}$, where the $\mathbb{Z}_{r}$ does not affect the $\mathrm{AdS}_{5} \times S^{1}$ factor. The dual geometry of any $\mathcal{N}=2$ superconformal theory has an $\operatorname{AdS}_{5} \times S^{1}$ factor, since the protected members of the $\mathcal{N}=2$ chiral ring precisely match the Kaluza-Klein reduction of the six-dimensional tensor multiplet on this $\operatorname{AdS}_{5} \times S^{1}$ factor $[8,15]$. Wilson loops provide a way to probe the dual geometry and, in particular, to measure the size of the $\operatorname{AdS}_{5} \times S^{1}$ factor because, on the string theory side, they are described by a minimal surface which classically ends on the contour of the Wilson loop. Calculating the expectation value of the circular Wilson loop on both sides of the correspondence

*mitev@math.hu-berlin.de

†elli.pomoni@desy.de has been one of the first successful tests of the $\mathcal{N}=4$ AdS/CFT paradigm $[16,17]$ and with this paper we begin a similar program for $\mathcal{N}=2$ theories.

In four dimensions, $\mathcal{N}=4 \mathrm{SYM}$ is the unique, up to a choice of the gauge group, maximally supersymmetric gauge theory, and it has exactly one marginal coupling constant. The space of conformal $\mathcal{N}=2$ gauge theories is classified by ADE [14,18-20] finite or affine Dynkin diagrams. By sending a coupling constant to zero, one can obtain the superconformal theories that correspond to the finite Dynkin diagrams from the affine ones. For simplicity, in the present article we will only consider the elliptic quivers based on the affine $\hat{A}_{r-1}$ Dynkin diagrams that can be obtained from $\mathbb{Z}_{r}$ orbifolds of $\mathcal{N}=4 \mathrm{SYM}$. The simplest example in this class is the $\mathbb{Z}_{2}$ elliptic quiver. This is the $\mathrm{SU}\left(N_{c}\right) \times \mathrm{SU}\left(N_{c}\right)$ theory with two marginal couplings $g, \check{g}$ which, in the limit $\check{g} \rightarrow 0$, leads to superconformal QCD (SCQCD) with color group $\mathrm{SU}\left(N_{c}\right)$ and $N_{f}=2 N_{c}$ flavor hypermultiplets that has been studied extensively in [8,21-25].

In [1] we show that the purely gluonic $\mathrm{SU}(2,1 \mid 2)$ sector of composite operators in every $\mathcal{N}=2$ theory, made out of fields only in the vector multiplet $\phi, \lambda_{+}^{\mathcal{I}}, \mathcal{F}_{++}, \mathcal{D}_{+\dot{\alpha}}$, is closed to all loops in planar perturbation theory. This sector includes operators that correspond to string states classically living only on the $\operatorname{AdS}_{5} \times S^{1}$ factor of the dual geometry. We also present a diagrammatic argument that anomalous dimensions in the $\mathrm{SU}(2,1 \mid 2)$ sector can be read off from the $\mathcal{N}=4$ ones up to a redefinition, due to finite renormalization, of the coupling constant $g^{2} \rightarrow f\left(g^{2}\right)$, i.e. 


$$
\gamma^{\mathcal{N}=2}\left(g^{2}\right)=\gamma^{\mathcal{N}=4}\left(f\left(g^{2}\right)\right), \quad \text { where } g^{2}=\frac{g_{\mathrm{YM}}^{2} N_{c}}{(4 \pi)^{2}} .
$$

Thus, we can use the integrability of planar $\mathcal{N}=4$ and the results available to compute the anomalous dimensions for planar $\mathcal{N}=2$ theories of operators in this sector, as long as we can compute the effective coupling $f\left(g^{2}\right)$.

In this paper we compute these functions for the $\hat{A}_{r-1}$ theories (15), and we interpret them as the relative finite renormalization of the coupling constant

$$
f\left(g^{2}\right)-g^{2}=g^{2}\left[\left(\mathcal{Z}_{g}^{\mathcal{N}=2}\right)^{2}-\left(\mathcal{Z}_{g}^{\mathcal{N}=4}\right)^{2}\right] .
$$

The calculation of the effective couplings is done via the evaluation of the expectation value of the circular Wilson loop. Using localization, Pestun was able to prove the conjecture of $[16,17]$ that the expectation values of the circular Wilson loops for any $\mathcal{N}=2$ theory can be obtained using matrix models [3]. Here, we use these matrix models to calculate the Wilson loop expectation values, and we show that

$$
\begin{aligned}
& W^{\mathcal{N}=2}\left(g^{2}\right)=W^{\mathcal{N}=4}\left(f\left(g^{2}\right)\right), \quad \text { with } \\
& W^{\mathcal{N}=4}\left(g^{2}\right)=\frac{I_{1}(4 \pi g)}{2 \pi g},
\end{aligned}
$$

where $I_{1}$ is the first modified Bessel function of the first kind. From Eqs. (1) and (3), we learn that the integrable $\mathcal{N}=4$ theory knows all about the combinatorics involved in the Feynman diagram calculations. To get to the $\mathcal{N}=2$ theory result, all we need to do is compute the relative finite renormalization of the coupling constant that is encoded in the effective coupling $f\left(g^{2}\right)$. On the dual gravity side, the effective couplings are interpreted as the renormalization of the effective string tension,

$$
T_{\mathrm{eff}}^{2}=\frac{R^{4}}{\left(2 \pi \alpha^{\prime}\right)^{2}}=f\left(g^{2}\right) .
$$

For the $\mathbb{Z}_{2}$ quiver, the first correction of the effective coupling $f\left(g^{2}\right)$ from the weak-coupling side was computed in $[23,26]$ using Feynman diagrams,

$$
f\left(g^{2}\right)=\left\{\begin{array}{ll}
g^{2}+12\left(\check{g}^{2}-g^{2}\right) \zeta(3) g^{4}+\cdots, & g, \check{g} \rightarrow 0 \\
2 \frac{g^{2} \check{g}^{2}}{g^{2}+\check{g}^{2}}+\cdots, & g, \check{g} \rightarrow \infty
\end{array},\right.
$$

while the first term of the strong-coupling expansion was written in [25] by using AdS/CFT. In Sec. III, we write the first few orders of the weak-coupling expansion of $f\left(g^{2}\right)$, discuss their Feynman diagram interpretation, and give the leading term in the strong-coupling limit.

\section{THE DIAGRAMMATIC ARGUMENT AND THE POWER OF GAUGE INVARIANCE}

Classical gauge theory has local gauge invariance which is broken by the addition of a gauge-fixing term during quantization. The background field formalism (BFF) provides a way to keep manifest as much as possible of the local gauge invariance. To use it, we separate the gauge field $A_{\mu}$ into a classical and a quantum part: $A_{\mu}=\mathcal{A}_{\mu}+\mathrm{Q}_{\mu}$. The bare and the renormalized quantities are related by the renormalization factors

$$
\begin{aligned}
\mathcal{A}_{\text {bare }}^{\mu} & =\sqrt{\mathcal{Z}_{\mathcal{A}}} \mathcal{A}_{\text {ren }}^{\mu}, \quad \mathrm{Q}_{\text {bare }}^{\mu}=\sqrt{\mathcal{Z}_{\mathrm{Q}}} \mathrm{Q}_{\text {ren }}^{\mu}, \\
g_{\text {bare }} & =\mathcal{Z}_{g} g_{\text {ren }}, \quad \xi_{\text {bare }}=\mathcal{Z}_{\xi} \xi_{\text {ren }},
\end{aligned}
$$

where $\xi$ is the gauge-fixing parameter. For simplicity, we present only the Yang Mills part of the theory, but the procedure carries over to quarks and also to supersymmetric $\mathcal{N}=1$ and $\mathcal{N}=2$ theories in the appropriate superspace [27-30].

In the background field gauge, the renormalization factors are related as

$$
\mathcal{Z}_{g} \sqrt{\mathcal{Z}_{\mathcal{A}}}=1, \quad \mathcal{Z}_{\mathrm{Q}}=\mathcal{Z}_{\xi},
$$

and the final answer for any gauge invariant quantity will only depend on the $\mathcal{Z}_{\mathcal{A}}$ factor. What is more, in the BFF the renormalization factors for the quantum fields $\mathcal{Z}_{Q}$ will cancel for each individual diagram. This can be easily seen by recalling a couple of BFF corollaries. In the BFF Feynman diagrams, the classical fields $\mathcal{A}_{\mu}$ cannot propagate on the internal lines. They only appear as external fields in correlation functions. Moreover, all offshell n-point functions $\left\langle\mathrm{Q}_{\mu_{1}} \cdots \mathrm{Q}_{\mu_{\ell_{1}}} \mathcal{A}_{\nu_{1}} \cdots \mathcal{A}_{\nu_{\ell_{2}}}\right\rangle$ renormalize as $\mathcal{Z}_{\mathrm{Q}}^{\ell_{1} / 2} \mathcal{Z}_{\mathcal{A}}^{\ell_{2} / 2} \mathcal{Z}_{g}^{n}\left\langle\mathrm{Q}_{\mu_{1}} \cdots \mathrm{Q}_{\mu_{\ell_{1}}} \mathcal{A}_{\nu_{1}} \cdots \mathcal{A}_{\nu_{\ell_{2}}}\right\rangle$. Finally, each internal propagator $\left\langle Q_{\mu} Q_{\nu}\right\rangle$ carries a factor of $\mathcal{Z}_{\mathrm{Q}}^{-1}$. Composite local or nonlocal operators like Wilson loops should be inserted in their renormalized form $\mathcal{O}_{i}^{\text {ren }}\left(Q_{\text {ren }}, \mathcal{A}_{\text {ren }}\right)=\sum_{j} \mathcal{Z}_{i j} \mathcal{O}_{j}^{\text {bare }}\left(\mathcal{Z}_{\mathrm{Q}}^{1 / 2} \mathrm{Q}, \mathcal{Z}_{\mathcal{A}}^{1 / 2} \mathcal{A}\right)$ where $\mathcal{Z}_{i j}$ is the the mixing matrix.

In [1] we presented a diagrammatic argument that for any planar and superconformal $\mathcal{N}=2$ theory, the asymptotic $\mathrm{SU}(2,1 \mid 2)$ Hamiltonian is identical to all loops to that of $\mathcal{N}=4$ SYM, up to a redefinition of the coupling constant $g^{2} \rightarrow f\left(g^{2}\right)$. Thus, this sector is integrable and anomalous dimensions can be read off from the $\mathcal{N}=4$ ones, up to this redefinition.

A refined version of the diagrammatic argument in [1] is reviewed below, based only on

(i) gauge invariance (background field method),

(ii) the chirality of the $\mathrm{SU}(2,1 \mid 2)$ sector which makes the non-renormalization theorem of $[31,32]$ applicable. 
To explain the argument, we begin by considering $\mathcal{N}=2$ theories obtained as orbifolds of $\mathcal{N}=4$ SYM. They are conformal by inheritance arguments [33,34]. When all the coupling constants are equal to each other (orbifold point), all anomalous dimensions in the untwisted sector are equal to the $\mathcal{N}=4$ ones.

In order to compute the renormalization of operators, we write down all the relevant diagrams and compute each one of them in $\mathcal{N}=4$ (at the orbifold point) as well as in $\mathcal{N}=2$ and subtract the results from each other. All the individual UV-divergent Feynman diagrams that should be calculated for the renormalization of operators in the $\mathrm{SU}(2,1 \mid 2)$ sector, are identical in both theories. The only diagrams that are different from their $\mathcal{N}=4$ counterparts are finite and they are responsible for the relative finite renormalization between the $\mathcal{N}=2$ and the $\mathcal{N}=4$ coupling constants. Some examples of such diagrams are depicted in Figs. 1, 2 and 3.

This procedure should be thought of as a novel regularization prescription that cancels the divergencies of each individual diagram. The fact that the difference of the two diagrams is always finite stems from the finiteness of the $\mathcal{N}=2$ theories we are considering [18] and from the fact that the purely gluonic tree level terms in both the $\mathcal{N}=2$ and the $\mathcal{N}=4$ Lagrangians are identical. With this powerful regularization prescription, we can simplify our computations. All the combinatorics and symmetry factors of the individual diagrams are identical in both theories. So, we let the $\mathcal{N}=4$ integrable model give them to us, and we just have to compute the difference (2).

There is one possible way this argument could fail. Going up to higher order in $g$, new nonlocal vertices will appear in the effective action of $\mathrm{N}=2$ theories that are not there for $\mathcal{N}=4 \mathrm{SYM}$. In $\mathcal{N}=2$ superspace language, we have the effective action $\Gamma=\int d^{4} \theta \mathcal{F}+$ c.c. $+\int d^{8} \theta \mathcal{H}$, see

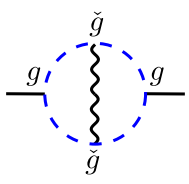

FIG. 1 (color online). The Feynman diagram responsible for the $\zeta(3)$ contribution to $f(g, \check{g})$. The solid lines represents the background $\mathcal{N}=2$ vector superfield, the wiggly line the quantum $\mathcal{N}=2$ vector superfield, and the dashed blue lines, the $\mathcal{N}=2$ hyperfield.

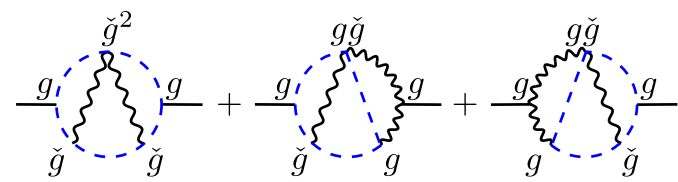

FIG. 2 (color online). In this figure we present some representative Feynman diagrams that are responsible for the $\zeta(5)$ contribution to $f(g, \breve{g})$.

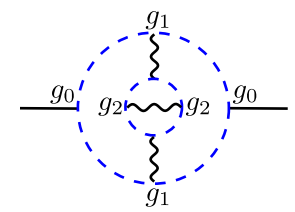

FIG. 3 (color online). This figure shows a part of the $\zeta(3)^{2}$ contribution created by nested Feynman diagrams.

[1]. However, none of these new nonholomorphic vertices (from $\mathcal{H}$ ) can contribute to the anomalous dimensions of the $\mathrm{SU}(2,1 \mid 2)$ sector [1] due to the nonrenormalization theorem of [31,32]. Only the renormalized tree level vertices from the holomorphic prepotential $\mathcal{F}$ will contribute. Due to the fact that the $\mathcal{Z}_{\mathrm{Q}}$ cancel, the final result depends only on $\mathcal{Z}_{\mathcal{A}}=\mathcal{Z}_{g}^{-2}$. Thus all anomalous dimensions obey $\gamma_{i}\left(g^{2}\right)=\gamma_{i}^{\mathcal{N}=4}\left(f\left(g^{2}\right)\right)$ with $f\left(g^{2}\right)$ given in (2). Equipped with this observation, we turn to Pestun's circular Wilson loop. Pestun's choice of localizing $Q$ makes manifest the fact that the Wilson loop receives contributions only from the holomorphic prepotential $\mathcal{F}$ and not from $\mathcal{H}$, thus depending only on one single $\mathcal{Z}$ factor (in the BFF) $\mathcal{Z}_{g}$.

\section{WILSON LOOPS}

Pestun's matrix models provide an efficient way to compute the expectation value of the circular Wilson loop,

$W_{k}^{\mathcal{N}=2}=\left\langle\frac{1}{N_{c}} \operatorname{tr}_{\square} \operatorname{Pexp} \oint_{\mathcal{C}} d s\left(i A_{\mu}^{(k)}(x) \dot{x}^{\mu}+\phi^{(k)}(x)|\dot{x}|\right)\right\rangle$,

where $\square$ denotes the fundamental representation and $\mathcal{C}$ is the circular loop located at the equator of $S^{4}$. The adjoint scalar $\phi^{(k)}$ and the gauge field $A_{\mu}^{(k)}$ are in the vector multiplet of the $k$ th gauge group. Inserting in the path integral a composite operator with fields only in the $k$ th vector multiplet selects the coupling $g_{k}^{2}$ whose renormalization we are computing,

$$
W_{k}^{\mathcal{N}=2}\left(g_{1}, \ldots, g_{r}\right)=W^{\mathcal{N}=4}\left(f_{k}\left(g_{1}, \ldots, g_{r}\right)\right),
$$

where $f_{k}\left(g_{1}, \ldots, g_{r}\right)=g_{k}^{2}+\cdots$ is the effective coupling constant of the $k$ th gauge group.

Let us consider a cyclic quiver made out of $r$ gauge groups, corresponding to the untwisted affine Dynkin diagram $\hat{A}_{r-1}$. We follow the method and notations of [35-38]. The partition function of the corresponding matrix model is 


$$
\begin{aligned}
Z= & \int \prod_{k=1}^{r} d a^{(k)} \prod_{i<j=1}^{N_{c}} \\
& \times\left(a_{i}^{(k)}-a_{j}^{(k)}\right)^{2} e^{-\frac{N_{c}}{2 g_{k}^{2}} \sum_{i=1}^{N_{c}}\left(a_{i}^{(k)}\right)^{2}} Z_{1 \text {-loop }}\left|Z_{\text {inst }}\right|^{2} .
\end{aligned}
$$

In the planar limit, the instanton contribution can be neglected, while the one loop part is

$$
Z_{\text {1-loop }}=\prod_{k, l=1}^{r} \prod_{i, j=1}^{N} H^{\frac{\mathbf{a}_{k l}}{2}}\left(a_{i}^{(k)}-a_{j}^{(l)}\right)
$$

where $H(x)=\prod_{n=1}^{\infty}\left(1+\frac{x^{2}}{n^{2}}\right)^{n} e^{-\frac{x^{2}}{n}}$ and $\mathbf{a}_{k l}$ is the Cartan matrix corresponding to $\hat{A}_{r-1}$. By using the saddle point approximation and replacing in the planar limit the eigenvalues $a_{i}^{(k)}$ by normalized densities $\rho_{k}(x)$ that are localized in an interval $\left[-\mu_{k}, \mu_{k}\right]$, we obtain the following system of coupled integral equations:

$$
\frac{x}{2 g_{k}^{2}}=f_{-\mu_{k}}^{\mu_{k}} \frac{\rho_{k}(y)}{x-y} d y-\frac{1}{2} \sum_{l=1}^{r} \mathbf{a}_{k l} \int_{-\mu_{l}}^{\mu_{l}} \rho_{l}(y) K(x-y) d y,
$$

for $k=1, \ldots, r$. For small values of the couplings, the widths of the densities tend towards zero and we can expand the kernel $K(x)=-2 \sum_{n=1}^{\infty}(-1)^{n} \zeta(2 n+1) x^{2 n+1}$. Then we can solve the integral equations recursively and compute the Wilson loop expectation values of Eq. (8) via

$$
W_{k}^{\mathcal{N}=2}=\left\langle\frac{1}{N_{c}} \sum_{i=1}^{N_{c}} e^{2 \pi a_{i}^{(k)}}\right\rangle=\int_{-\mu_{k}}^{\mu_{k}} \rho_{k}(x) e^{2 \pi x} d x
$$

For the elliptic $\mathbb{Z}_{2}$ quiver with couplings $g_{1}=g, g_{2}=\check{g}$, we obtain

$$
\begin{aligned}
f(g, \check{g})= & g^{2}+2\left(\check{g}^{2}-g^{2}\right)\left[6 \zeta(3) g^{4}-20 \zeta(5) g^{4}\left(\check{g}^{2}+3 g^{2}\right)\right. \\
& +g^{4}\left(70 \zeta(7)\left(\check{g}^{4}+5 \check{g}^{2} g^{2}+8 g^{4}\right)-2 \zeta(2)(20 \zeta(5)) g^{4}\right. \\
& \left.\left.-2(6 \zeta(3))^{2}\left(\check{g}^{4}-\check{g}^{2} g^{2}+2 g^{4}\right)\right)\right]+\cdots
\end{aligned}
$$

Inserting the above in $W^{\mathcal{N}=4}(f(g, \check{g}))$ and taking the limit $\check{g} \rightarrow 0$, we recover the $\mathcal{N}=2$ SCQCD computation of [35]. For the general superconformal cyclic $\hat{A}_{r-1}$ quivers, we obtain up to order $\mathcal{O}\left(g^{10}\right)$,

$$
\begin{aligned}
f_{k}= & g_{k}^{2}+6 \zeta(3) g_{k}^{4}\left[g_{k-1}^{2}+g_{k+1}^{2}-2 g_{k}^{2}\right]-20 \zeta(5) g_{k}^{4}\left[g_{k-1}^{4}+g_{k+1}^{4}-6 g_{k}^{4}+2 g_{k}^{2}\left(g_{k-1}^{2}+g_{k+1}^{2}\right)\right] \\
& +g_{k}^{4}\left[70 \zeta(7)\left(g_{k-1}^{6}+g_{k+1}^{6}-16 g_{k}^{6}+3 g_{k}^{4}\left(g_{k-1}^{2}+g_{k+1}^{2}\right)+4 g_{k}^{2}\left(g_{k-1}^{4}+g_{k+1}^{4}\right)\right)-2 \zeta(2)(20 \zeta(5)) g_{k}^{4}\left(g_{k-1}^{2}+g_{k+1}^{2}-2 g_{k}^{2}\right)\right. \\
& \left.+(6 \zeta(3))^{2}\left(8 g_{k}^{6}-2 g_{k-1}^{6}-2 g_{k+1}^{6}+g_{k-1}^{4} g_{k-2}^{2}+g_{k+2}^{2} g_{k+1}^{4}-6 g_{k}^{4}\left(g_{k-1}^{2}+g_{k+1}^{2}\right)+2 g_{k}^{2}\left(g_{k-1}^{4}+g_{k-1}^{2} g_{k+1}^{2}+g_{k+1}^{4}\right)\right)\right]+\cdots,
\end{aligned}
$$

while the leading term at strong coupling is

$$
f_{k}=r \frac{g_{1}^{2} \cdots g_{r}^{2}}{\sum_{i=1}^{r} \prod_{j \neq i} g_{j}^{2}}+\cdots
$$

which agrees with the AdS/CFT prediction $[8,14,25]$. The $\mathbb{Z}_{r}$ symmetry implies the following cyclic relation,

$$
f_{k}\left(g_{1}, \ldots, g_{r}\right)=f_{k+l}\left(g_{1+l}, \ldots, g_{r+l}\right), \quad \forall k, l \text {; }
$$

i.e., all the effective couplings are given by the same function, up to a cyclic shift of the couplings.

\section{FEYNMAN DIAGRAM INTERPRETATION}

Calculating $f\left(g^{2}\right)$ using Feynman diagrams is not as hard as one would imagine because of its interpretation as the relative finite renormalization of the coupling constant (2). First of all, in the BFF one does not have to calculate the renormalization of 3- or 4-point vertices as with usual covariant gauges, but to use $\mathcal{Z}_{g}=\mathcal{Z}_{\mathcal{A}}^{-1 / 2}$ and to compute only the renormalization of the propagator $\langle\mathcal{A}(p) \mathcal{A}(-p)\rangle$. Moreover, to get (2), we do not need to calculate every single diagram that contributes to the renormalization of the propagator, but only the ones that are different between $\mathcal{N}=2$ and $\mathcal{N}=4$ (or the orbifold of $\mathcal{N}=4$ at the orbifold point). As we discussed in [1,23], for any $\mathcal{N}=2$ superconformal theory the only possible way to get diagrams different from the $\mathcal{N}=4$ ones is to make a loop with hypermultiplets and to let a vector field from a neighboring vector multiplet propagate inside this loop. This narrows down significantly the number of Feynman diagrams that need to be computed.

It so happens that the type of diagrams that are different from the $\mathcal{N}=4$ ones are always finite, and they always include as a basic building block the finite fan integrals of [39]. For a fan with $n$ faces, we have

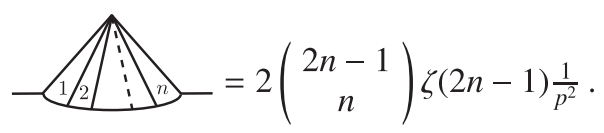

The first $\zeta(3)$ contribution in (14) was computed in [23], comes from the diagram depicted in Fig. 1, and is equal to $12 g^{4} g^{2} \zeta(3)$. Subtracting from it the $\mathcal{N}=4$ result of $12 g^{6} \zeta(3)$ gives precisely the $\zeta(3)$ coefficient in (14). 
The next correction comes with a $\zeta(5)$ and is obtained from diagrams, of different graph topologies, in which two propagators are running in the bubble. For each graph topology, there are three diagrams. In Fig. 2, we give an example of the diagrams with the same topology. Their sum is proportional to $g^{4} g^{4}+2 g^{2} g^{6}$, which happens to be the same for all topologies. After subtracting the $\mathcal{N}=4$ result, which is proportional to $3 g^{8}$, from the $\mathcal{N}=2$ one, we

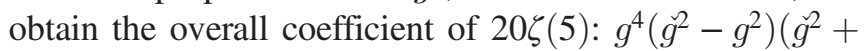
$\left.3 g^{2}\right)$ which is equal to the one in (14). Observe that the overall sign of the $\zeta(2 n-1)$ contribution is alternating, because each wiggly line comes with a minus sign.

All fan diagrams come with maximum transcendentality for the given loop level. However, as we see in (14) and (15), less than maximum transcendentality contributions can appear. These come from nested diagrams like the one depicted in Fig. 3. For the general cyclic quiver with $r>2$, we start getting contributions from the next-to-nearestneighbor gauge groups for the $\zeta(3)^{2}$ term of (15).

Finally, the origin of the $\zeta(2) \zeta(5)$ contribution has not been fully elucidated. We believe that it is due to scheme dependence originating from the fact that the localization computation is done on the sphere and not in flat space. A careful calculation is in progress.

\section{DISCUSSION AND FUTURE WORK}

Building on [1], we have argued that the anomalous dimensions of operators in the purely gluonic $\mathrm{SU}(2,1 \mid 2)$ sector of conformal $\mathcal{N}=2$ gauge theories can be obtained by taking the corresponding $\mathcal{N}=4$ result and replacing the $\mathcal{N}=4$ coupling constant with the effective coupling $f\left(g^{2}\right)$. Localization provides exact results for the expectation values of circular Wilson loops, from which we determine the weak-coupling expansion as well as the leading strongcoupling term of the effective couplings. Finally, we interpreted $f\left(g^{2}\right)$ diagrammatically as the relative finite renormalization of the coupling constant between the $\mathcal{N}=2$ and the $\mathcal{N}=4$ theories (2). This provides a test of our results using Feynman diagrams.

Based on the existence of an AdS dual description of these $\mathcal{N}=2$ gauge theories and on the interpretation of the effective couplings as the effective string tensions $T_{\text {eff }}^{2}=f\left(g^{2}\right)$, we conjecture that all possible observables that are restricted to the purely gluonic $\mathrm{SU}(2,1 \mid 2)$ sector can be computed by replacing the $\mathcal{N}=4$ coupling constant in the corresponding results with the universal effective coupling $f\left(g^{2}\right)$. Such observables include the cusp anomalous dimension [40] and the Bremsstrahlung function [41] (see also [42] for recent results) as well as lightlike Wilson loops (see [43] and references therein) and correlation functions $[44,45]$.

In a future work, we will present similar results for the asymptotically conformal quiver theories in which conformality is softly broken by adding mass terms for the hypermultiplets. While our methods are applicable and $g^{2}$ is again corrected only with finite renormalization, understanding the scheme dependence is subtle and requires further investigation.

One way to test our results for the effective couplings is to consider the anomalous dimension of the twist-two descendant of Konishi. Using the anomalous dimension of Konishi in $\mathcal{N}=4$ SYM, which thanks to integrability is known up to eight loops [46], we can predict the anomalous dimension of the twist-two $D^{2} Z^{2}$ descendant to the same loop order for any of the cyclic quivers. This prediction can be explicitly checked by computing the wrapping corrections using Feynman diagram calculations to at least four loops following [47]. To conserve space, we restrict ourselves to five loops and to the $\hat{A}_{1}$ quiver. The contributions to $\Delta$ that differ from the $\mathcal{N}=4$ ones are marked in bold:

$$
\begin{aligned}
& \Delta(g, \check{g})=4+12 g^{2}-48 g^{4}+48 g^{4}\left[7 g^{2}-\mathbf{3}\left(\boldsymbol{g}^{\mathbf{2}}-\boldsymbol{g}^{\mathbf{2}}\right) \zeta(\mathbf{3})\right]+96 g^{4}\left[-26 g^{4}+6 \zeta(3) g^{4}-15 \zeta(5) g^{4}+\left(\mathbf{g}^{\mathbf{2}}-\boldsymbol{g}^{\mathbf{2}}\right)\left(\mathbf{1 2} \boldsymbol{g}^{\mathbf{2}} \zeta(\mathbf{3})\right.\right. \\
& \left.\left.+\mathbf{5}\left(\mathbf{3} \boldsymbol{g}^{2}+\boldsymbol{g}^{\mathbf{2}}\right) \zeta(\mathbf{5})\right)\right]+16 g^{4}\left[948 g^{6}+432 g^{6} \zeta(3)-324 g^{6} \zeta(3)^{2}-540 g^{6} \zeta(5)\right. \\
& +1890 g^{6} \zeta(7)-3\left(g^{2}-g^{2}\right)\left[\left(8 g^{4}+5 g^{2} g^{2}+g^{4}\right) 35 \zeta(7)\right. \\
& \left.\left.-g^{2}\left(4 g^{2}+g^{2}(12-\zeta(2))\right) 20 \zeta(5)-\left(2 g^{4}-g^{2} g^{2}+g^{4}\right)(6 \zeta(3))^{2}+42 g^{4}(6 \zeta(3))\right]\right]+\cdots \text {. }
\end{aligned}
$$

Our results can also be used for observables outside the $\mathrm{SU}(2,1 \mid 2)$ sector. The all-loop dispersion relation and scattering matrix for fields in the bifundamental hypermultiplet in the $\phi$ vacuum were derived in [22]

$$
E_{\mathrm{bif}}(p)=\sqrt{1+4(\mathbf{g}-\check{\mathbf{g}})^{2}+16 \mathbf{g} \mathbf{g s i n}^{2}\left(\frac{p}{2}\right)},
$$

and up to two unknown functions that we conjecture are given by the effective couplings $\mathbf{g}=f(g, \check{g})^{\frac{1}{2}}$ and $\check{\mathbf{g}}=\check{f}(g, \check{g})^{\frac{1}{2}}$ that we calculated (14) in this paper. Due to the $\mathbb{Z}_{2}$ symmetry, we have $\check{f}(g, \check{g})=f(\check{g}, g)$. Thus the dispersion and the scattering matrix are now exactly known.

Our work is the four-dimensional equivalent of the result of [48] for the ABJM theory [49], even though our methods are very different. The quantum spectral curve and the slope functions of Basso [50] can be used to check our logic. Furthermore, the results for the interpolating function $h_{A B J M}(g)$ [48] can be combined with our method to derive $h_{A B J}(g)$ of the ABJ theory [51]. 
The present paper contains the principles regarding the computation of the effective couplings via localization as well as their Feynman diagram interpretation. In a forthcoming publication, we shall provide additional details and give expressions concerning the strong-coupling expansion and the implementation of mass terms for the hypermultiplets.

\section{ACKNOWLEDGMENTS}

We are grateful to Wenbin Yan for giving us his unpublished notes on the calculation of the Wilson loop of the $\mathbb{Z}_{2}$ quiver using localization. We thank Isabella Bierenbaum, Sergey Frolov, Sven-Olaf Moch, Leonardo Rastelli, Martin Roček, Alessandro Sfondrini, Christoph Sieg, and Peter van Nieuwenhuizen for insightful and helpful discussions.
[1] E. Pomoni, Nucl. Phys. B893, 21 (2015).

[2] N. Beisert, C. Ahn, L. F. Alday, Z. Bajnok, J. M. Drummond et al., Lett. Math. Phys. 99, 3 (2012).

[3] V. Pestun, Commun. Math. Phys. 313, 71 (2012).

[4] O. Aharony, S. S. Gubser, J. M. Maldacena, H. Ooguri, and Y. Oz, Phys. Rep. 323, 183 (2000).

[5] M. Grana and J. Polchinski, Phys. Rev. D 65, 126005 (2002).

[6] H. Lin, O. Lunin, and J. M. Maldacena, J. High Energy Phys. 10 (2004) 025.

[7] D. Gaiotto and J. Maldacena, J. High Energy Phys. 10 (2012) 189.

[8] A. Gadde, E. Pomoni, and L. Rastelli, arXiv:0912.4918.

[9] R. Reid-Edwards and B. Stefanski, Nucl. Phys. B849, 549 (2011).

[10] E. O Colgain and B. Stefanski, J. High Energy Phys. 10 (2011) 061.

[11] O. Aharony, L. Berdichevsky, and M. Berkooz, J. High Energy Phys. 08 (2012) 131.

[12] B. Stefanski, Nucl. Phys. B883, 581 (2014).

[13] S. Kachru and E. Silverstein, Phys. Rev. Lett. 80, 4855 (1998).

[14] A. E. Lawrence, N. Nekrasov, and C. Vafa, Nucl. Phys. B533, 199 (1998).

[15] S. Gukov, Phys. Lett. B 439, 23 (1998).

[16] J. Erickson, G. Semenoff, and K. Zarembo, Nucl. Phys. B582, 155 (2000).

[17] N. Drukker and D. J. Gross, J. Math. Phys. (N.Y.) 42, 2896 (2001).

[18] P. S. Howe, K. Stelle, and P. C. West, Phys. Lett. B 124, 55 (1983).

[19] S. Katz, P. Mayr, and C. Vafa, Adv. Theor. Math. Phys. 1, 53 (1998).

[20] N. Nekrasov and V. Pestun, arXiv:1211.2240.

[21] A. Gadde, E. Pomoni, and L. Rastelli, J. High Energy Phys. 06 (2012) 107.

[22] A. Gadde and L. Rastelli, J. High Energy Phys. 04 (2012) 053.

[23] E. Pomoni and C. Sieg, arXiv:1105.3487.

[24] P. Liendo, E. Pomoni, and L. Rastelli, J. High Energy Phys. 07 (2012) 003.

[25] A. Gadde, P. Liendo, L. Rastelli, and W. Yan, J. High Energy Phys. 08 (2013) 015.
[26] R. Andree and D. Young, J. High Energy Phys. 09 (2010) 095.

[27] S. Gates, M. T. Grisaru, M. Rocek, and W. Siegel, Front. Phys. 58, 1 (1983).

[28] I. Buchbinder, E. Buchbinder, S. Kuzenko, and B. A. Ovrut, Phys. Lett. B 417, 61 (1998).

[29] I. L. Buchbinder and S. M. Kuzenko, Mod. Phys. Lett. A 13, 1623 (1998).

[30] D. Jain and W. Siegel, Phys. Rev. D 88, 025018 (2013).

[31] F. Fiamberti, A. Santambrogio, C. Sieg, and D. Zanon, Nucl. Phys. B805, 231 (2008).

[32] C. Sieg, Phys. Rev. D 84, 045014 (2011).

[33] M. Bershadsky, Z. Kakushadze, and C. Vafa, Nucl. Phys. B523, 59 (1998).

[34] M. Bershadsky and A. Johansen, Nucl. Phys. B536, 141 (1998).

[35] F. Passerini and K. Zarembo, J. High Energy Phys. 09 (2011) 102.

[36] J. Russo and K. Zarembo, J. High Energy Phys. 10 (2012) 082.

[37] J. Russo and K. Zarembo, J. High Energy Phys. 11 (2013) 130.

[38] J. Russo and K. Zarembo, arXiv:1312.1214.

[39] D. J. Broadhurst, Phys. Lett. B 164, 356 (1985).

[40] G. Korchemsky, Lett. Math. Phys. 99, 425 (2012).

[41] D. Correa, J. Henn, J. Maldacena, and A. Sever, J. High Energy Phys. 06 (2012) 048.

[42] B. Fiol, E. Gerchkovitz, and Z. Komargodski, arXiv: 1510.01332.

[43] S. Caron-Huot, J. High Energy Phys. 07 (2011) 058.

[44] B. Eden, P. Heslop, G. P. Korchemsky, and E. Sokatchev, Nucl. Phys. B869, 329 (2013).

[45] B. Eden, P. Heslop, G. P. Korchemsky, and E. Sokatchev, Nucl. Phys. B869, 378 (2013).

[46] S. Leurent and D. Volin, Nucl. Phys. B875, 757 (2013).

[47] F. Fiamberti, A. Santambrogio, C. Sieg, and D. Zanon, Phys. Lett. B 666, 100 (2008).

[48] N. Gromov and G. Sizov, Phys. Rev. Lett. 113, 121601 (2014).

[49] https://inspirehep.net/record/787590.

[50] B. Basso, arXiv:1109.3154.

[51] O. Aharony, O. Bergman, and D. L. Jafferis, J. High Energy Phys. 11 (2008) 043. 